\title{
Seroprevalencia de infección por Helicobacter pylori en población adulta de Lima, Perú 2017
}

Arturo Pareja Cruz ${ }^{1, a}$, Pedro Javier Navarrete Mejía ${ }^{2, a}$, José Francisco Parodi García ${ }^{\text {3,a }}$

RESUMEN

Objetivo: Conocer la seroprevalencia de infección por Helicobacter pylori en población adulta de Lima, Perú 2017.

Materiales y métodos: Estudio descriptivo, prospectivo, transversal. Población conformada por voluntarios mayores de 18 años, de ambos sexos, con o sin molestias gastroenterológicas generales. Campaña de despistaje realizada en los distritos de Magdalena y Chorrillos de la ciudad de Lima, Perú en el mes de enero del 2017. Para el diagnóstico se utilizó la prueba rápida OnSite $\mathrm{H}$. pylori Ab Combo Rapid Test CE de CTK Biotech.

Resultados: Se evaluó a 140 pacientes, edad media 36.6 años, $22.1 \%$ de sexo masculino y $77.9 \%$ de sexo femenino. La seroprevalencia para Helicobacter pylori fue $63.6 \%$.

Conclusiones: Nosotros concluimos que la infección por Helicobacter pylori es frecuente en el área de la ciudad de Lima, sin diferencia entre género y edad.

Palabras clave: Helicobacter pylori; cáncer de estómago; gastritis; úlcera gástrica.

\section{Seroprevalence of Helicobacter pylori infection among an adult population of Lima, Peru 2017}

\section{ABSTRACT}

Objective: To know the seroprevalence of Helicobacter pylori infection among an adult population of Lima, Peru 2017. Materials and methods: Descriptive, prospective, cross-sectional study. Population of volunteers older than 18 years, of both sexes, with or without general gastrointestinal discomfort. A screening campaign was carried out in the districts of Magdalena and Chorrillos in the city of Lima, Peru, in January 2017. For the diagnosis, CTK Biotech's OnSite H. pylori Ab Combo Rapid Test CE was used.

Results: One hundred forty (140) patients were evaluated, with a mean age of 36.6 years old, being $22.1 \%$ male and $77.9 \%$ female. The seroprevalence of Helicobacter pylori infection was $63.6 \%$.

Conclusions: We conclude that Helicobacter pylori infection is common in the city of Lima, with no difference between gender and age.

Keywords: Helicobacter pylori; stomach neoplasms; gastritis; stomach ulcer.

1. Maestro en Salud Pública y Gestión de Sistemas de Salud, Responsable del Centro de Investigación en Infectología e Inmunología.

2. Doctor en Salud Pública, Responsable del Centro de Investigación de Salud Pública.

3. Maestro en Salud Pública, Responsable del Centro de Investigación en Envejecimiento.

a. Instituto de Investigación, Facultad de Medicina Humana, Universidad de San Martín de Porres. Lima, Perú. 


\section{INTRODUCCIÓN}

El Helicobacter pylori es una bacteria Gram negativa, pequeña y espiralada que habita en la mucosa gástrica de humanos. Desde su descubrimiento por Warren y Marshall, se la ha asociado a casos de gastritis activa, enfermedad ulcerosa péptica, linfoma MALT y en los últimos años una fuerte asociación con el cáncer gástrico, siendo esta bacteria catalogada desde 1991 como un agente carcinogénico ${ }^{(1-3)}$.

La prevalencia mundial se ha estimado alrededor del $50 \%$ siendo esta cifra mucho mayor en países en vías de desarrollo superando el $80 \%$ de la población. En nuestro país, estudios realizados al respecto dan una cifra similar a la literatura internacional sobre la prevalencia en países en vías de desarrollo oscilando este dato entre el $50 \%$ y $70 \%$. Su transmisión es fecal-oral y se asocia al mal nivel sanitario en una población, así como una pobre higiene, la cual favorece que se concentre cifras altas de prevalencia ${ }^{(4-13)}$.

El cáncer gástrico es la tercera causa de muerte por cáncer en todo el mundo, y la infección por Helicobacter pylori causa alrededor del $90 \%$ de los cánceres fuera del cardias. La infección por $\mathrm{H}$. pylori es tratable, y en los ensayos clínicos hay evidencia de una reducción del $30 \%$ al $40 \%$ de la incidencia de cáncer gástrico entre los sujetos tratados. Sin embargo, salvo algunas excepciones, no existen programas de salud pública para prevención del cáncer gástrico ${ }^{(14,15)}$.

En diciembre de 2013, la Agencia para la Investigación del Cáncer (IARC), organizó un Grupo de Trabajo de expertos internacionales para debatir y formular recomendaciones sobre el control del cáncer gástrico. El grupo de trabajo consideró que una enorme carga de enfermedades, no se espera que disminuya las próximas décadas, para lo cual se requiere una acción decisiva de salud pública que incluya el cáncer gástrico en los programas de control del cáncer ${ }^{(16)}$.

Las intervenciones deben adaptarse a las condiciones locales y considerar la posibilidad de detección y erradicación de $\mathrm{H}$. pylori, en el contexto de evaluación de la viabilidad, la eficacia y las consecuencias adversas ${ }^{(17)}$.

En nuestro país, la prevención de enfermedades asociadas al $\mathrm{H}$. pylori debe constituir un sistema de desarrollo de prevención sostenible que contemple la detección precoz y oportuna de este agente infeccioso; la alta significancia de este aspecto en la salud pública hace que se requiera vigilar la prevalencia de $\mathrm{H}$. pylori en nuestra población mediante nuevas técnicas rápidas y confiables de inmunodiagnóstico que ofrezca un perfil de seroprevalencia en la población peruana.

\section{MATERIALES Y MÉTODOS}

Estudio descriptivo, prospectivo, transversal. Diseño no experimental.

La población estuvo conformada por voluntarios de ambos sexos mayores de 18 años con o sin molestias gastroenterológicas generales. El recojo de información se realizó en una campaña médica realizada en dos distritos de Lima metropolitana; distrito de Chorrillos (cobertura pobladores del este y sur de Lima) y distrito de Magdalena del Mar (cobertura pobladores del centro y norte de Lima). Se firmaron los consentimientos respectivos.

El método de diagnóstico utilizada fue la prueba de inmunoserología rápida por la técnica de inmunocromatografía de flujo lateral, la prueba permite la detección en forma conjunta IgM, IgG e IgA; para la muestra se utilizó una gota de sangre periférica capilar de pulpejo de dedo.

Se utilizó el Kit de prueba rápida On Site H. pylori" Ab CE de CTK Biotech. Sensibilidad $93.55 \%$, especificidad $96.94 \%$. Los resultados se presentan haciendo uso de estadística descriptiva.

\section{RESULTADOS}

Se evaluaron a 140 pacientes, la edad media de la población es estudio fue 36.6 años; el $22.1 \%$ de los pacientes fueron de sexo masculino y el $77.9 \%$ de sexo femenino.

Los grupos etarios de mayor frecuencia fueron los comprendidos entre 21 y 60 años de edad (85.7\%).

La mayor seroprevalencia por grupo de edad se determinó para los voluntarios $<=20$ años $(75.0 \%)$ y $31-40$ años $(67.9 \%)$. La prevalencia por sexo fue mayor en el sexo femenino (65.1\%).

La seroprevalencia de Helicobacter pylori para la población total estudiada fue $63.6 \%$ (Tabla 1).

Tabla 1. Seroprevalencia de Helicobacter pylori. Lima, Perú. 2017

\begin{tabular}{rrrr}
\multicolumn{2}{c}{$\begin{array}{c}\text { Característica } \\
\text { Sexo }\end{array}$} & Frecuencia & Positivos (\%) \\
& Masculino & 31 & $18(58.0 \%)$ \\
Edad & Femenino & 109 & $71(65.1 \%)$ \\
& & & \\
& $<=20$ & 4 & $3(75.0 \%)$ \\
& $\mathbf{2 1 - 3 0}$ & 35 & $23(65.7 \%)$ \\
& $\mathbf{3 1 - 4 0}$ & 28 & $19(67.9 \%)$ \\
& $\mathbf{4 1 - 5 0}$ & 30 & $20(66.7 \%)$ \\
& $\mathbf{5 1 - 6 0}$ & 27 & $17(63.0 \%)$ \\
& $\mathbf{6 1 - 7 0}$ & 9 & $4(44.4 \%)$ \\
& $>70$ & 7 & $3(42.9 \%)$ \\
Total & $>30$ & 39 & $26(66.7 \%)$ \\
& $>30$ & 101 & $63(62.4 \%)$ \\
& & 140 & $89(63.6 \%)$
\end{tabular}

Fuente: Elaboración propia 


\section{DISCUSIÓN}

Cada año se diagnostican en el planeta casi 13 millones de casos de cáncer - cifra ascendente año a año -, evidenciándose que aún tenemos problemas en su control; la OMS estima que para el año 2030 se habrán presentado más de 20 millones de casos nuevos, entre el 2005 y 2015 han fallecido por esta enfermedad más de 90 millones de personas ${ }^{(18)}$.

En los países en vías de desarrollo el problema del cáncer se agudiza más; la población tiene bajos ingresos, estilos de vida no saludables y los recursos para diagnóstico y prevención son limitados; la OPS calcula que un tercio de las muertes por cánceres se produce en América Latina ${ }^{(19)}$.

El disponer de agua potable no es suficiente en la prevención de la infección por Helicobacter pylori, hoy se tiene evidencia que la bacteria resiste al cloro; la Universidad Johns Hopkins demostró que el agua potable que beben los pobladores de Lima presentaba material genético de la bacteria Helicobacter pylori ${ }^{(20,21)}$.

La infección por Helicobacter pylori constituye un factor de riesgo para cáncer de estómago, este tipo de cáncer representa la principal causa de mortalidad por cáncer en el Perú. La evidencia científica demuestra que aproximadamente el $60 \%$ de la población mundial, en algún momento de su vida, se infecta por esta bacteria y entre el $1,3 \%$ de ellos desarrollara cáncer de estómago a futuro ${ }^{(22,23)}$

En el caso del Perú, el incremento de los casos del cáncer se puede explicar por aspectos relacionados a la transición demográfica, pobreza, estilo de vida, género, raza, entre los más importantes. De acuerdo a la Vigilancia Epidemiológica de Cáncer del Ministerio de Salud, el 14.9\% son cáncer de cérvix, $11.1 \%$ de estómago, $10.3 \%$ de mama, $6.6 \%$ de piel y $5.8 \%$ de próstata; el cáncer constituye el $8.0 \%$ de la carga de enfermedad nacional ${ }^{(21)}$. Pilco y Col ya reportaban que, en Lima, hace más de una década, el cáncer gástrico había aumentado desde el año 1990 hasta 1997, llegando a tener 24,3/100000 en hombres, y en mujeres 17,6/100000, en el último periodo estudiado, considerándose área de alto riesgo ${ }^{(25)}$.

El año 2012 en el Perú, como parte de la política pública para hacer frente al cáncer, se desarrolló como estrategia de atención integral y mejoramiento de acceso a los servicios oncológicos el Plan Esperanza; el interés nacional es que la población de menores recursos pueda acceder a servicios de calidad y por qué no decirlo, generar una mejor vigilancia epidemiológica del cáncer.

En razón de una relación entre infección por $\mathrm{H}$. pylori y cáncer, según concluye la reunión de la IARC en diciembre de 2013, la detección temprana en los países es necesaria, así como crear planes de prevención contra el cáncer gástrico e intervención contra la infección por H. pylori. ${ }^{(24)}$.

La investigación determinó una seroprevalencia general de 63.6\% para los pobladores de la ciudad de Lima en Perú; este valor de seroprevalencia es cercana a lo reportado en México con un $52.2 \%(8)$, y $52.9 \%$ en Corea ${ }^{(7)}$, pero menor comparado con la seroprevalencia determinada en China $(83.4 \%)^{(6)}$ y reportado en Etiopía $(83.3 \%)^{(11)}$. En un estudio en nuestro medio, Ramírez Ramos y Col, luego de evaluar 1815 endoscopías realizadas entre 1985 y el 2002, en pacientes de nivel socioeconómico medio y alto, encontró una disminución significativa de la prevalencia de la infección del estómago por H. pylori: 83,3\% en 1985, $75,1 \%$ en $1990,65,0 \%$ en 1996 , y $58,7 \%$ en el $2002^{(26)}$. Soto y $\mathrm{col}^{(27)}$ estudiaron a un grupo de pacientes de nivel socioeconómico bajo en las Pampas de San Juan, Lima, confirmando la alta prevalencia reportada en este estrato social hace una década (mayor al $90,0 \%)^{(28)}$.

Los hallazgos sugieren una mayor prevalencia de la infección en el grupo etario de 21 a 60 años de edad $(85.7 \%)$. Sin embargo, se ha reportado que la infección en nuestro medio se adquiere a edades tempranas de la vida. La forma de transmisión más importante probablemente sea la fecal-oral y a través del agua. Dados los riesgos ya descritos que esta conlleva, y usando el enfoque de curso de vida acciones adecuadas para su control podrían representar un ejemplo de cómo intervenciones en una etapa de vida pueden traducirse en éxito en otra etapa ${ }^{(29)}$.

Con respecto a la prevalencia según sexo, investigaciones en Alemania y China demostraron una mayor seroprevalencia en los pacientes de sexo masculino evaluados, $45.1 \%$ y $84.7 \%$ respectivamente ${ }^{(5,6)}$.

En conclusión, la infección por Helicobacter pylori, presenta una seroprevalencia similar a otras investigaciones, pero no debemos soslayar la asociación entre la bacteria y otras patologías; la importancia desde el punto de vista de salud pública debiera permitir generar estrategias de prevención temprana, más aún cuando el Perú presenta al cáncer de estómago como la más frecuente en su población masculina. La infección por Helicobacter pylori es frecuente en el área de la ciudad de Lima, para la población en estudio, sin diferencia entre género y edad.

Se deben desarrollar más investigaciones con métodos sencillos, válidos y de bajo costo como el propuesto para cubrir los datos de prevalencia a la infección en el resto del país y así guiar la gestión adecuada de las intervenciones.

\section{REFERENCIAS BIBLIOGRÁFICAS}

1. Castillo O, Maguiña J, Benites H, Chacaltana A, Guzmán E, Dávalos M, et al. Prevalence of Helicobacter pylori in symptomatic outpatients in Red Rebagliati (EsSalud) from 2010 to 2013, Lima, Perú. Rev Gastroenterol Perú. 2016;36(1):4955.

2. Barreda C, Arcana R, Salazar F, Barriga E, Monge V, Salazar F, et al. Lower prevalence of Helicobacter pylori infection observed in patients with erosive esophagitis. Rev Gastroenterol Peru. 2014; 34(1):33-7.

3. Prochazka R, Salazar F, Barriga E, Salazar F. Prevalence of Helicobacter pylori in a private practice setting in Lima, Peru. Sensitivity of biopsies and rapid urease test. Rev Gastroenterol Peru. 2010; 30(1):33-9. 
4. Rocha G, Oliveira A, Queiroz D, Mendes E, Moura S, Oliveira C, et al. Serodiagnosis of Helicobacter pylori infection by Cobas Core ELISA in adults from Minas Gearais, Brazil. Braz J Med Biol Res 1998; 31(10): 1263-68.

5. Wex T, Venerito M, Kreutzer J, Götze T, Kandulski A, Malfertheiner P. Serological Prevalence of Helicobacter pylori Infection in Saxony-Anhalt, Germany, in 2010.Clin Vaccine Inmunol. 2011; 18(12): 2109-12.

6. Zhang $M$, Zhou $Y$, Li X, Tang Z, Zhu H, Yang $Y$, et al. Seroepidemiology of Helicobacter pylori infection in elderly people in the Beijing región, china. World J Gastroentrol. 2014; 20(13): 3635-39.

7. Kim H, Kim N, Kim S, Seo J, Park E, Lee D. Seroprevalence of Helicobacter pylori infection in Korean Health Personnel. Gut and Liver. 2013; 7(6): 648-54.

8. Alvarado-Esquivel C. Seroepidemiology of Helicobacter pylori infection in Pregnant Women in Rural Durango, Mexico. Int J Biomed Sci. 2013; 9(4): 224-29.

9. Camargo M, Beltran M, Conde-Glez C, Harris P, Michel A, Waterboer T, et al. Serological response to Helicobacter pylori infection among Latin American populations with contrasting risk of gastric cáncer. Int J Cancer. 2015; 137: 3000-5.

10. Alvarado-Esquivel C. Seroepidemiology of Helicobacter pylori in Tepehuanos Aged 15 Years and Older in Durango, Mexico. Journal of Pathogens. 2013; 1 -5.

11. Tadesse E, Daka D, Yemane D, Shimelis T. Seroprevalence of Helicobacter pylori infection and related risk factors in symptomatic patients in southern Ethiopia. BMC Research Notes. 2014; 7(1): 834-38.

12. Jaka H, Mushi M, Mirambo M, Wilson L, Seni J, Mtebe $M$, et al. Sero-prevalence and associated factors of Helicobacter pylori infection among adult patient with dispepsia attending the gastroenterology unit in a tertiary hospital in Mwanza, Tanzania. African Health Sciences. 2016; 16(3): 684-9.

13. Kishikawa H, Kimura K, Takarabe S, Kaida S, Nishida J. Helicobacter pylori Antibody Titer and Gastric Cancer Screening. Disease Markers. 2015; 1-11.

14. Lozano-Pope I, Sharma A, Matthias M, Doran K, Obonyo M. Effect of myeloid differentiation primary response gene 88 on expression profiles of genes during the development and progression of Helicobacter-induced gastric cancer. BMC Cancer. 2017; 17(1):133.

15. Burkitt M, Duckworth C, Williams J, Pritchard D. Helicobacter pylori-induced gastric pathology: insights from in vivo and ex vivo models. Dis Model Mech. 2017; 10(2):89-104.

16. Herrero R, Park J, Forman D. The fight againts gastric cáncer - the IARC Working Group report. Best Practice \& Research Clinical Gastroenterology. 2014; 28(6): 1107-14.

17. Koczula K, Gallotta A. Lateral flow assays. Essays in Biochemistry. 2016; 60(1): 111-20.

18. Organización Panamericana de la Salud. El cáncer en la región de las Américas. OPS; 2012. Disponible en: http:// new. paho.org/hq/index.php?option=com_docman\&task=doc_ view\&gid $=16805 \&$ Itemid $=$.

19. Secretaría de Salud. Registro Histopatológico de Neoplasias Malignas. Dirección General Adjunta de Epidemiología. México, 1993-2004.
20. Uemura N, Okamoto S, Yamamoto S, Matsumura N, Yamaguchi $S$, Yamakido $M$, et al. Helicobacter pylori infection and the development of gastric cancer. N Engl J Med. 2001; 345(11):784-9.

21. Sachs G, Scott DR. Helicobacter pylori: Eradication or Preservation. F1000 Med Rep. 2012; 4(7):7-12

22. Ramírez-Ramos A, Sánchez-Sánchez R. Helicobacter pylori 25 años después (1983 -2008): Epidemiología, Microbiología, Patogenia, Diagnóstico y Tratamiento. Rev Gastroenterol Peru. 2009; 29(2):158-70.

23. Ramírez A, Chinga E, Mendoza D. Variación de la prevalencia del $\mathrm{H}$. pylori y su relación con los niveles de cloro en el agua de la Atarjea, Lima, Perú. Período 1985-2002. Rev Gastroent Perú. 2004;24(3):223-9.

24. Herrero R, Park J, Forman D. The fight against gastric cancer - the IARC Working Group report. Best Pract Res Clin Gastroenterol. 2014;28(6):1107-14.

25. Pilco C, Payet M, Cáceres G. Cáncer Gástrico en Lima Metropolitana. Rev Gastroent Perú. 2006;26(4):377-85.

26. Ramírez Ramos A, Chinga E, Mendoza D, Leey J, Guerra J. Estudio del Helicobacter pylori en el Perú. Rev Per Med Exp Salúd Pública. 2002;19(4):209-214. (En prensa)

27. Soto G, Bautista C, Roth D, Gilman R, Velapatiño B, Ogura M, et al. Helicobacter pylori reinfection is common in peruvian adults following successful antibiotic eradication therapy. J Infect Dis.2003; 188 (9): 1263-75

28. Ramirez-Ramos A, Gilman R, Spira W, Recavarren S, Watanabe $\mathrm{J}$, Leon-Barua R, et al. Ecology of Helicobacter pylori in Peru: infection rates in coastal, high altitude, and jungla communities. Gut. 1992; 33(5): 604-5.

29. Ramírez Ramos A, Mendoza Requena D, Leey Casella J, Guerra Valencia J. Estudio del Helicobacter pylori en el Perú. Rev. Perú. med. exp. salud pública.2002; 19(4):209-14.

\section{Fuentes de financiamiento:}

Este artículo ha sido financiado por los autores.

\section{Conflictos de interés:}

Los autores declaran no tener ningún conflicto de interés.

\section{Correspondencia:}

Arturo Pareja Cruz

Dirección: Av. Alameda del Corregidor 1531. Urb. Los Sirius. Las

Viñas, La Molina.

Teléfono: 998782686

Correo electrónico: aparejac@usmp.pe

Recibido: 24 de febrero de 2017 Aprobado: 16 de marzo de 2017 